Interestingly disease severity does not correlate with BD whereas more functional markers of frailty such as HGS appear to positively correlate. Increased disease severity (MELD) significantly correlates with decreasing vitamin D levels, which raises the question of whether vitamin $\mathrm{D}$ could be impacting on progression of cirrhosis, or vice versa. Further prospective research is needed to look at the role of vitamin $\mathrm{D}$ in cirrhosis.

\section{PTH-104 THE INCREASING BURDEN OF DIABETES ON THE SCOTTISH LIVER TRANSPLANTATION SERVICE}

Alexander Robertson*, Peter Hayes. Department of Gastroenterology, Royal Infirmary of Edinburgh, Edinburgh, UK

\subsection{6/gutjnl-2018-BSGAbstracts.260}

Introduction In Scotland $65 \%$ of adults are overweight and $29 \%$ obese $^{1}$. Among the health implications is a rising prevalence of diabetes within the population. Diabetes is known to contribute to liver damage, including carcinogenesis, and this study seeks to establish the impact of this damage on liver disease, hepatocellular carcinoma (HCC) and need for transplantation within Scotland. Diabetes, as a major risk factor for non-alcoholic fatty liver disease (NAFLD) could also be considered a surrogate marker for the NAFLD component of liver damage in other primary aetiologies.

Methods Prospectively held patient data from all transplant assessments undertaken at the Scottish liver transplant unit (SLTU) since 1992 were retrospectively analysed for the presence of diabetes, HCC and the underlying aetiology of their liver disease. Data were collected through VitalData $\left({ }^{\circ}\right.$ Vitalpulse Ltd) and exported into Microsoft Excel ( $\left({ }^{\odot}\right.$ Microsoft) for analysis.

Results In 1993 there were 45 assessments, which rose to 214 assessments in 2015, giving a total of 3098 during this time frame (figure 1).

The incidence of diabetes in patients referred for liver transplant remained persistently bellow 5\% until 2004, from which point it started to increase to $24 \%$ in 2016 (figure 2).

Figure 3 shows the percentages of patients who are referred for transplant assessment with HCC who were diabetic at the time of referral.

$12.0 \%$ of the 3098 patients referred had diabetes, this varied depending on their underlying aetiology- NAFLD (41.9\%), ALD (13.5\%), cryptogenic cirrhosis (9.6\%), HCV (9.2\%) and PBC (2.6\%). The percentage of patients with diabetes and HCC referred was $18.5 \%$ (of 574 patients)- NALFD (45.7\%), haemochromatosis (37.2\%), ALD (19.1\%), PSC (16.7\%), HBV (12.5\%), HCV (9.9\%) and others (0\%) (figure 4).

Conclusions The number of assessments has been increasing since the beginning of transplantation at the SLTU in 1992 with an increasing proportion diabetic. The impact is most clear through the trend in NAFLD reaching transplantation but the prevalence in other aetiologies of liver damage suggests a compound effect.

With the exception of PBC, each of the most common aetiologies seen showed a higher prevalence of diabetes in the patients referred with HCC. This suggests that diabetes promotes carcinogenesis, with the more modest impact in HCV mirroring previous studies into the carcinogenicity of diabetes $^{2}$

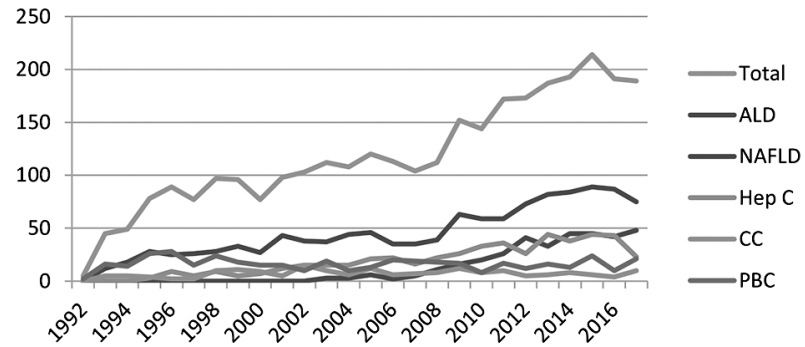

Abstract PTH-104 Figure 1 Total number of transplant assessments undertaken at SLTU

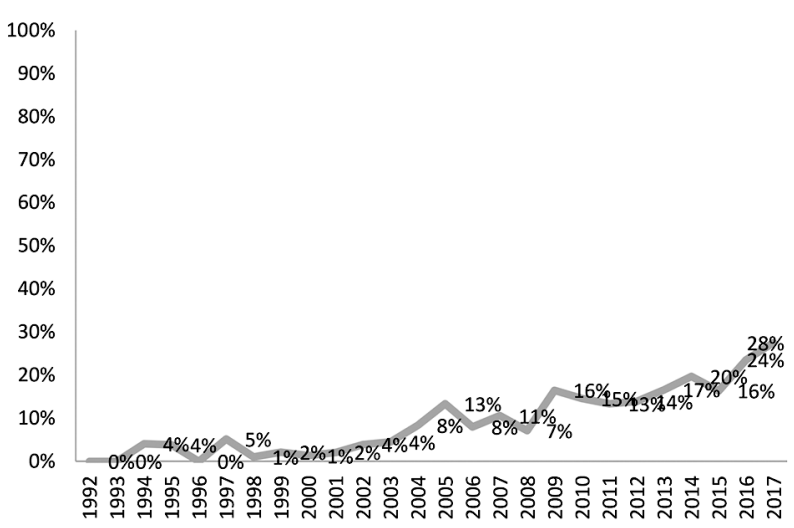

Abstract PTH-104 Figure 2 Percentage of patients assessed for transplant who have diabetes

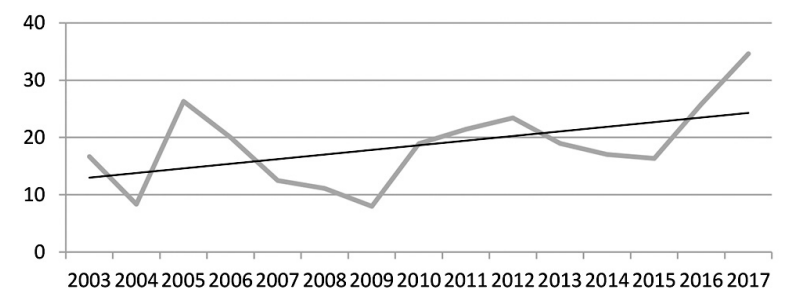

Abstract PTH-104 Figure 3 Percentage of patients referred for transplant assessment with HCC who were diabetic (by year)

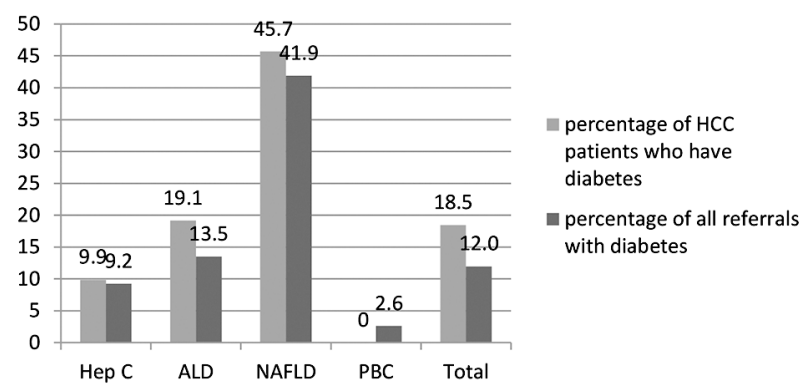

Abstract PTH-104 Figure 4 Percentages of referrals with HCC versus all referrals for each aetiology for transplant assessment

The increasing incidence of diabetes in patients being referred for transplant assessment with decompensated liver disease or HCC suggests that the national burden of diabetes and NAFLD ${ }^{3}$ will contribute to an increasing burden of liver damage of almost any aetiology. 